
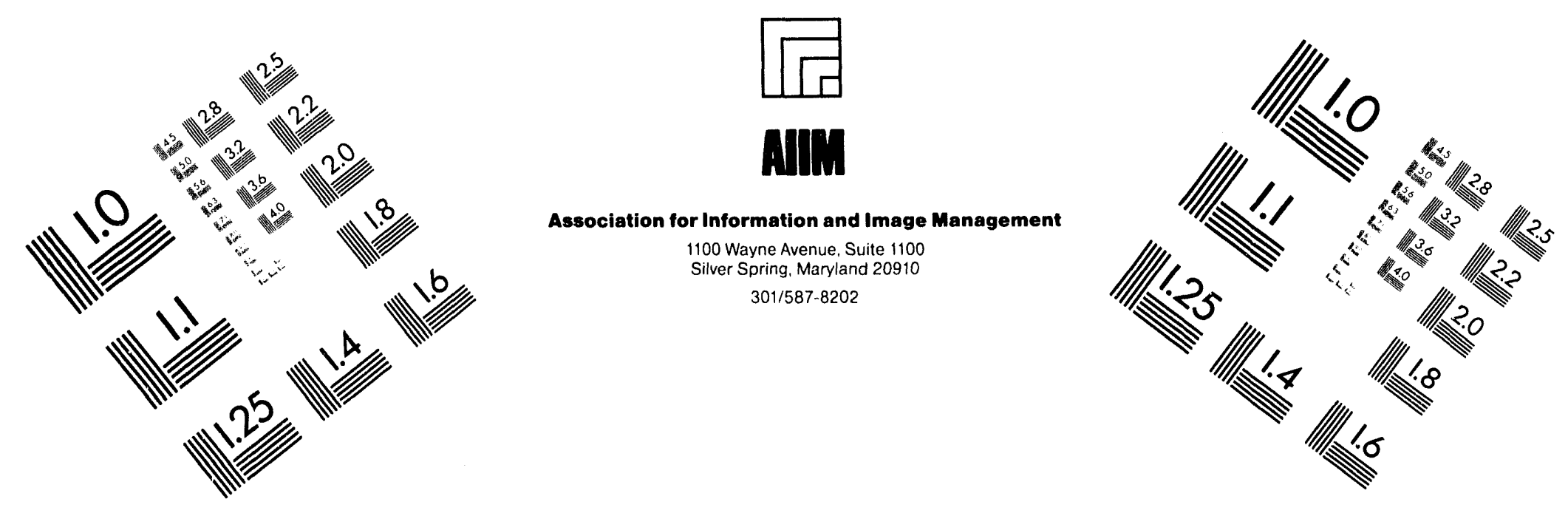

Centimeter

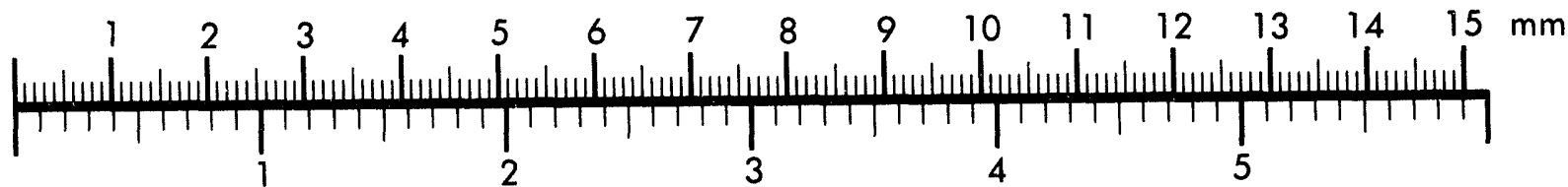
Inches
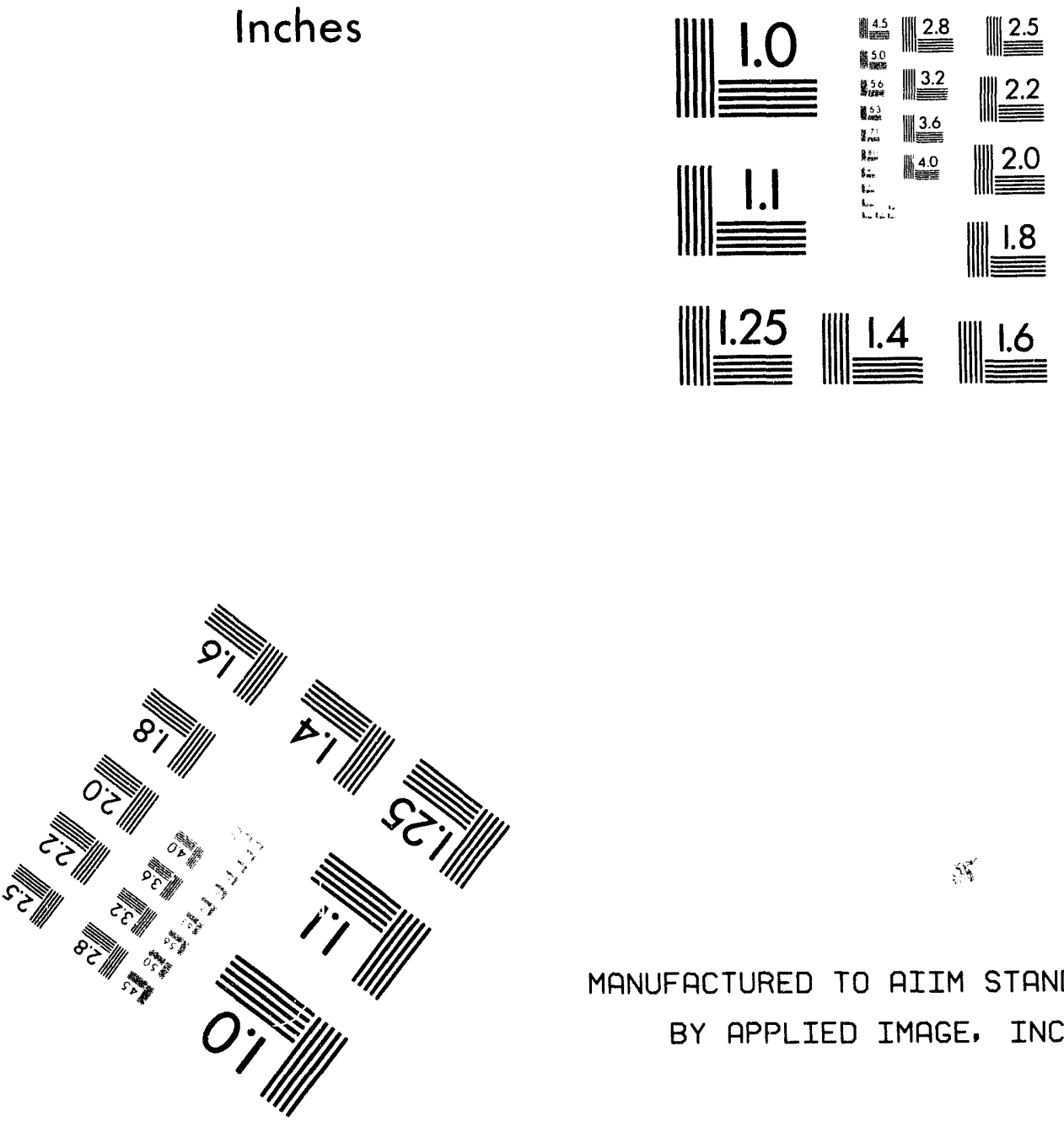

MANUFACTURED TO AIIM STANDARDS

BY APPLIED IMAGE, INC.

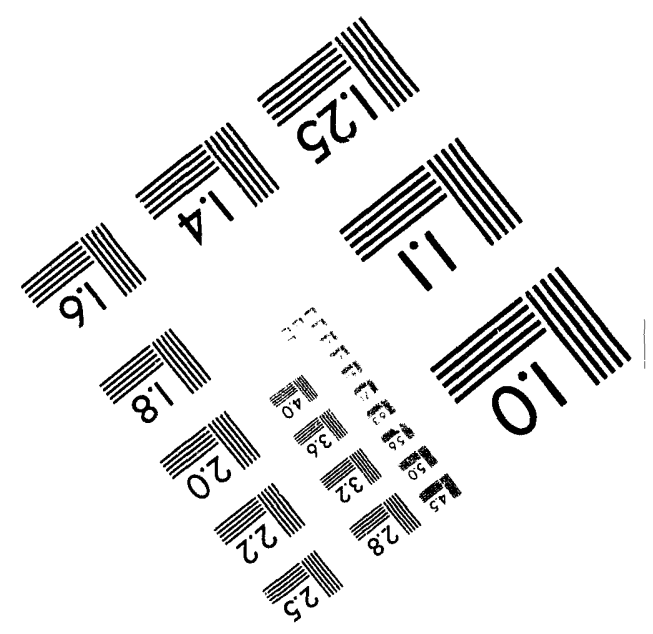



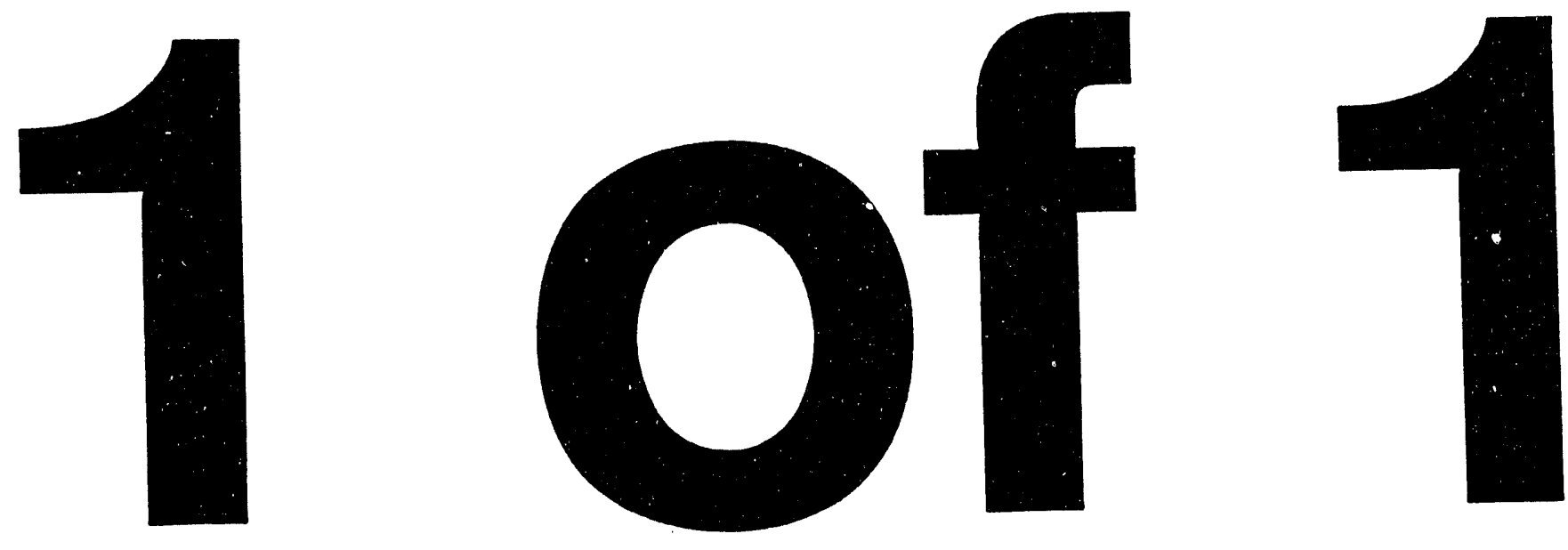


\title{
A Passive Scheme for ID End Correction
}

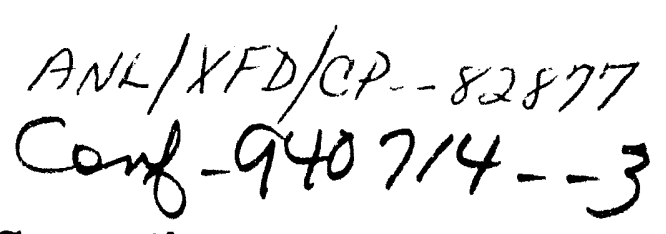

I. Vasserman and E.R. Moog

Advanced Photon Source, Argonne National Laboratory

9700 S. Cass Ave Argonne, IL 60439

\begin{abstract}
A passive end correction scheme used on a prototype undulator is described. This scheme allows the integrated field requirements to be met at all gaps from $11.5 \mathrm{~mm}$ to $200 \mathrm{~mm}$, with no active correction. The main parameters that need to be adjusted are the strength of the end magnets and the height of the next to last poles. The first field integral remains constant over the entire gap range, to within $25 \mathrm{G}-\mathrm{cm}$ in the vertical direction and $50 \mathrm{G}-\mathrm{cm}$ in the horizontal direction. The second field integral remains constant to within 10,000 G-cm 2 . Results of magnetic measurements are presented.
\end{abstract}

\section{DISCLAIMER}

This report was prepared as an account of work sponsored by an agency of the United States Government. Neither the United States Government nor any agency thereof, nor any of their employees, makes any warranty, express or implied, or assumes any legal liability or responsibility for the accuracy, completeness, or usefulness of any information, apparatus, product, or process disclosed, or represents that its use would not infringe privately owned rights. Reference herein to any specific commercial product, process, or service by trade name, trademark, manufacturer, or otherwise does not necessarily constitute or imply its endorsement, recommendation, or favoring by the United States Government or any agency thereof. The views and opinions of authors expressed herein do not necessarily state or reflect those of the United States Government or any agency thereof.

\section{MASTER}

DISTRIBUTION OF THIS DOCUMENT IS UNLIMITED 


\section{Introduction}

The Advanced Photon Source (APS), a 7-GeV positron storage ring, will have as many as 34 straight sections available for installation of multiple insertion devices (IDs). The size and the taper of the gap of the IDs will be able to be varied so that the energy of the emitted radiation can be tuned to the particular experiment. The IDs must not affect the particle beam motion elsewhere in the storage ring. Therefore, the disturbances should be kept within tight boundaries to assure that the ring performance parameters are maintained and that the emitted radiation shows little degradation from an ideal device.

The wedge-pole prototype hybrid undulator was designed and is being used to find the simplest, most reliable and least expensive way of satisfying the requirements imposed on IDs by the storage ring. The original wedge-pole prototype undulator design had a symmetric configuration for the ends; however, the requirement for gap independence of the first field integral through the undulator was not met. The following passive schemes for undulator end correction were tried, and they improved the magnetic field quality of the undulator:

1. An antisymmetric end configuration, 1,2

2. Half-strength magnets as the last magnets at each end of the device, and

3. Recess of the next- to-last pole tip. 3,4

A configuration was found that more than satisfies the requirements for the onaxis field gap dependence throughout the gap region from $11.5 \mathrm{~mm}$ to $200 \mathrm{~mm}$. In the following sections, details of the end adjustments are given.

We will not discuss here the horzontal field integrals or their correction, because they arise from imperfections in the magnetic structure rather than being inherent to the magnetic design of the insertion device and its ends. 


\section{Antisymmetric Configuration}

In an antisymmetric configuration, there is an even number of poles and the first pole at the upstream end of the undulator produces a field that is equal in magnitude but opposite in sign to that produced by the last pole at the downstream end, as shown in Fig. 1. Such a configuration results in a first field integral through the undulator that is inherently zero, because of the cancellation between the upstream and downstream ends. Some care still must be taken in the design of the ends to minimize the second field integral, but a great advantage of an antisymmetric end configuration is that the first and second integrals of the field can be adjusted independently by alterations in the ends. In a symmetric configuration, however, both integrals change when an alteration is made to the ends.

Figure 2 shows particle trajectories that were obtained from the second integrals of the Hall probe measurements. The trajectories shown have been averaged over one period length so as to eliminate the periodic wiggles; the effect can be seen by comparing Fig. 2(b) with its unaveraged version, which is shown as the trajectory in Fig. 1. Figure 2(a) shows the trajectory for the original symmetric configuration wit out any adjustment. The corresponding unadjusted trajectory for an antisymmetric configuration is shown in Fig. 2 (b).

\section{Reduced-Strength Magnets and Pole Recess}

Although using an antisymmetric configuration lessens the effect that the undulator has on the stored beam by making the exit trajectory parallel to the entrance trajectory (i.e., the first field integral through the undulator is zero), 
more remains to be corrected. As can he seen in Fig. 2(b), there is still a large offset in the trajectory at each end of the undul ator, with a slope to the trajectory through the periodic center part of the undulator. The offset and slope both vary with changes in the undulator gap and can cause unacceptably large variations in the trajectory over the full range of gaps, as shown in Fig. 3 by the curves labeled 'A'. (For purposes of comparison, the best result obtained for the symmetric configuration, even after some shimming of the end poles, was a variation of the first field integral by about $200 \mathrm{G}-\mathrm{cm}$ over the full range of gaps.) To minimize the gap dependence of the first and second integrals through the undulator, we therefore seek to minimize both the offset at the ends and the slope through the center section.

The first correction that was made was to eliminate the slope in the trajectory through the center of the undulator in order to decrease the gap dependence of the first field integral. In Fig. 1 it can be seen that the slope in the trajectory is in the direction opposite to the initial deflection of the stored beam by the first edge of the magnetic field. One would guess from this that correction of the slope would require either strengthening the field from the last pole or weakening the field from the next-to-last pole. Both were tried and both helped; the best results were obtained from weakening the next-to-last pole's field by grinding back the tip of that pole (effectively increasing the magnetic gap for that pole only). The curves labeled 'B' in Fig. 3, and labeled 'Full-strength end magnets' in Fig. 2(c) show the result of removing $1 \mathrm{~mm}$ from the pole tip. As can be seen in Fig. 2(c), the remaining trajectory displacement is due to the offset at the ends, at least at this gap. (A large offset with no slope for one gap will result in a different offset and some slope at another gap.) The variation of the overall trajectory displacement with gap is shown as curve 'B' in Fig. 3. 
The next correction that was made was to reduce the trajectory offset at the ends of the undulator. This was done by reducing the strength of the last magnet in the hybrid structure to about half the strength of the magnets in the rest of the undulator. As can be seen in Fig. 2(c), the trajectory is now near ideal. The importance of this reduced-strength magnet in minimizing the net trajectory displacement for an antisymmetric configuration can also be seen in Fig. 2(c). For an undulator with symmetric ends, the trajectory displacement at one end of the undulator is canceled by an opposite displacement at the other end, whereas for an antisymmetric undulator the displacements add.

The best configuration for this prototype undulator was found to be one in which the last magnets at each end were only half the strength of the rest of the magnets, and the next-to-last poles at each end were ground back by $0.5 \mathrm{~mm}$. This gives a pole strength pattern that is very close to the theoretical $1 / 4 \quad 3 / 4 \quad 1$ pattern that gives no displacement of the center of the trajectory in the undulator, and no net deflection or displacement of the trajectory after passage through the undulator. 5

The gap dependence of the first and second field integrals is shown in Fig. 3 as the curves labeled ' $\mathrm{C}$ '. The measurements of the first integral were made using a long moving coil; those of the second integral were obtained from Hall probe measurements.

There is an additional advantage to the use of reduced-strength magnets at the ends of the undulator. This is shown in Fig. 4, where the midplane magnetic field is plotted as a function of $\mathrm{z}$ for the prototype undulator at a large gap of 160 $\mathrm{mm}$. When full-strength magnets are used at the ends, there is still a relatively large field from the ends of the undulator even when the gap is so large that the periodic component of the field is too small to measure. When half-strength magnets are used at the ends, this end field is markedly reduced. 
We note that our primary goal in this work was to minimize the variation of the first and second field integrals with gap. A somewhat different optimization might be preferred for insertion devices that are not many periods long, when maximizing the number of full-field periods is of paramount importance.

\section{Conclusion}

A scheme has been found for adjusting the ends of an undulator so as to minimize the net effect that passage through the undulator has on the stored beam. This scheme has the added advantage of minimizing the effect throughout the range of undulator operating gaps, so that no active end correction is required. The scheme is based on an antisymmetric end configuration, with halfstrength magnets as the last magnets at each end of the undulator, and with the next-to-last pole tips at each end of the undulator recessed so those poles are effectively at a larger gap than the rest.

This work was supported by the U.S. Department of Energy, BES-Materials Sciences, under contract No. W-31-109-ENG-38. 


\section{REFERENCES}

1. L. Burkel, R. Dejus, J. Maines, J. O'Brien, J. Pflüger, and I. Vasserman, "The Insertion Device Magnetic Measurement Facility: Prototype and Operational Procedures," Argonne Nätional Laboratory Report ANL/APS/TB-12 (March 1993).

2. I. Vasserman, J. Pflüger, and E. Gluskin, "Magnetic Measurements of the Wedge-Pole Prototype Undulator," Argonne National Laboratory, Advanced Photon Source Report LS-222 (June 1993).

3. I. Vasserman, "Magnetic Measurements of the Wedge-Pole Prototype Undulator," in the report of the International Workshop on Magnetic Measurements of Insertion Devices, held Sept. 28-29, 1993 at Argonne National Laboratory, published as Argonne National Laboratory Report ANL/APS/TM-13 (October 1993), p. 102.

4. I. Vasserman and E. R. Moog, "A Passive Scheme for Undulator End Correction," Appendix 2 of the Technical Specification for Undulator A, Advanced Photon Source Document No. 41010101-00002.

5. K. Halbach, private communication. 


\section{CAPTIONS}

Fig. 1. (a) Hall probe measurements of the magnetic field in the undulator midplane as a function of position along the undulator's length, for an undulator gap of $11.5 \mathrm{~mm}$. (b) First integral of the field as a function of position. (c) Second integral of the field, or particle trajectory through the undulator.

Fig. 2. Particle trajectories, calculated from Hall probe data, for the prototype undulator with: (a) a symmetric end configuration, (b) an antisymmetric end configuration, and (c) an antisymmetric end configuration with the tips of the next-to-last poles at each end of the undulator recessed. The two cases shown in panel (c) are for configurations in which 1) the last magnets at each end of the undulator are full strength and there is a pole tip recess of $1 \mathrm{~mm}$, and 2) the magnets are half strength and there is a 0.5 mm pole tip recess. In panels (a) and (b) the magnets are full strength.

Fig. 3 Gap dependence of the first and second vertical field integrals for an antisymmetric configuration. (a) No end adjustment has been done. (b) The next-to-last poles at both ends of the undulator have their tips recessed by $1 \mathrm{~mm}$. (c) Pole tips are recessed by $0.5 \mathrm{~mm}$ and the last magnets at both ends of the undulator are half-strength.

Fig. 4 Magnetic field along the undulator, with the gap wide open at $160 \mathrm{~mm}$. The curve with the large peaks is for a configuration with full-strength magnets; the lower peak-field data is for a configuration with halfstrength magnets. 

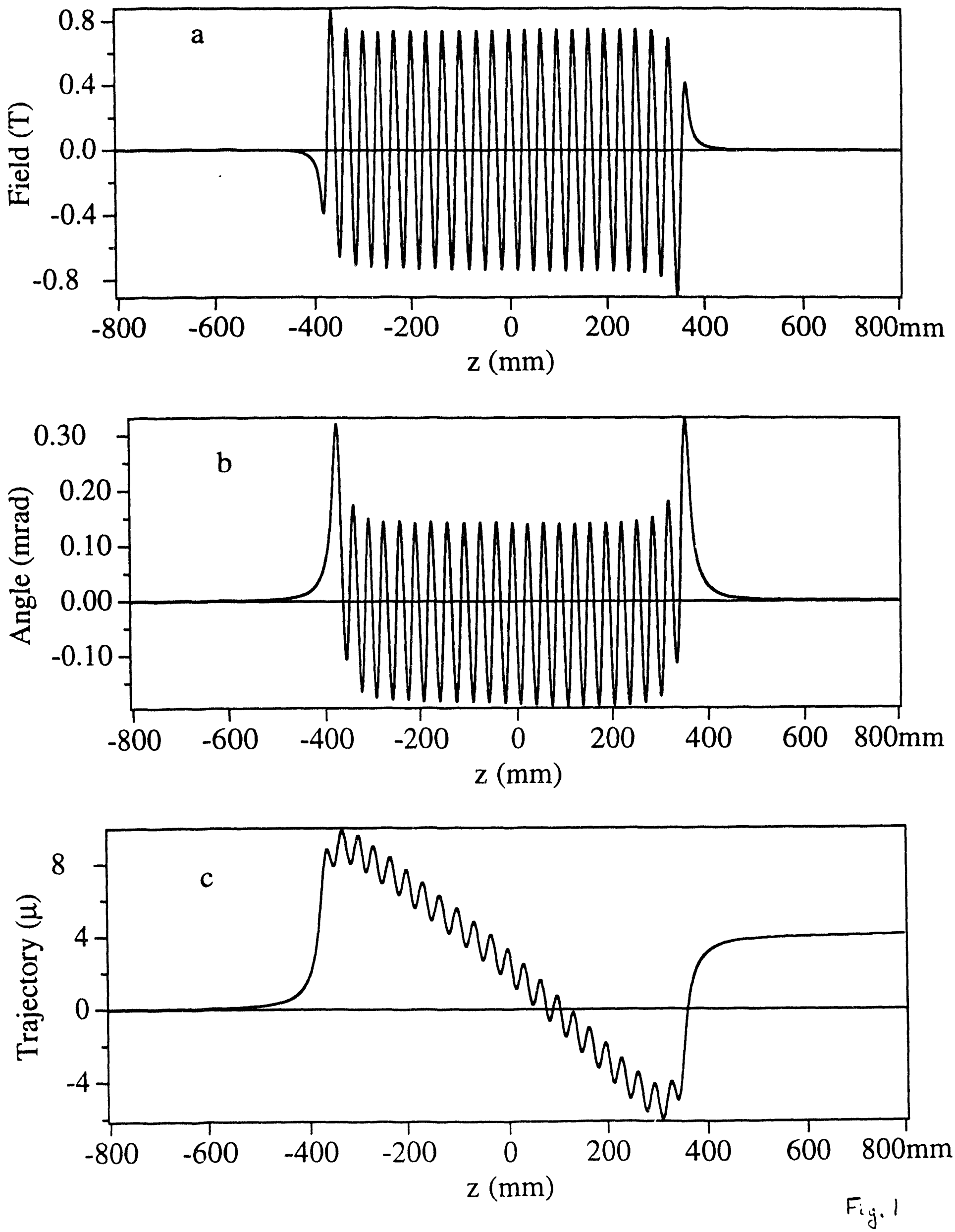

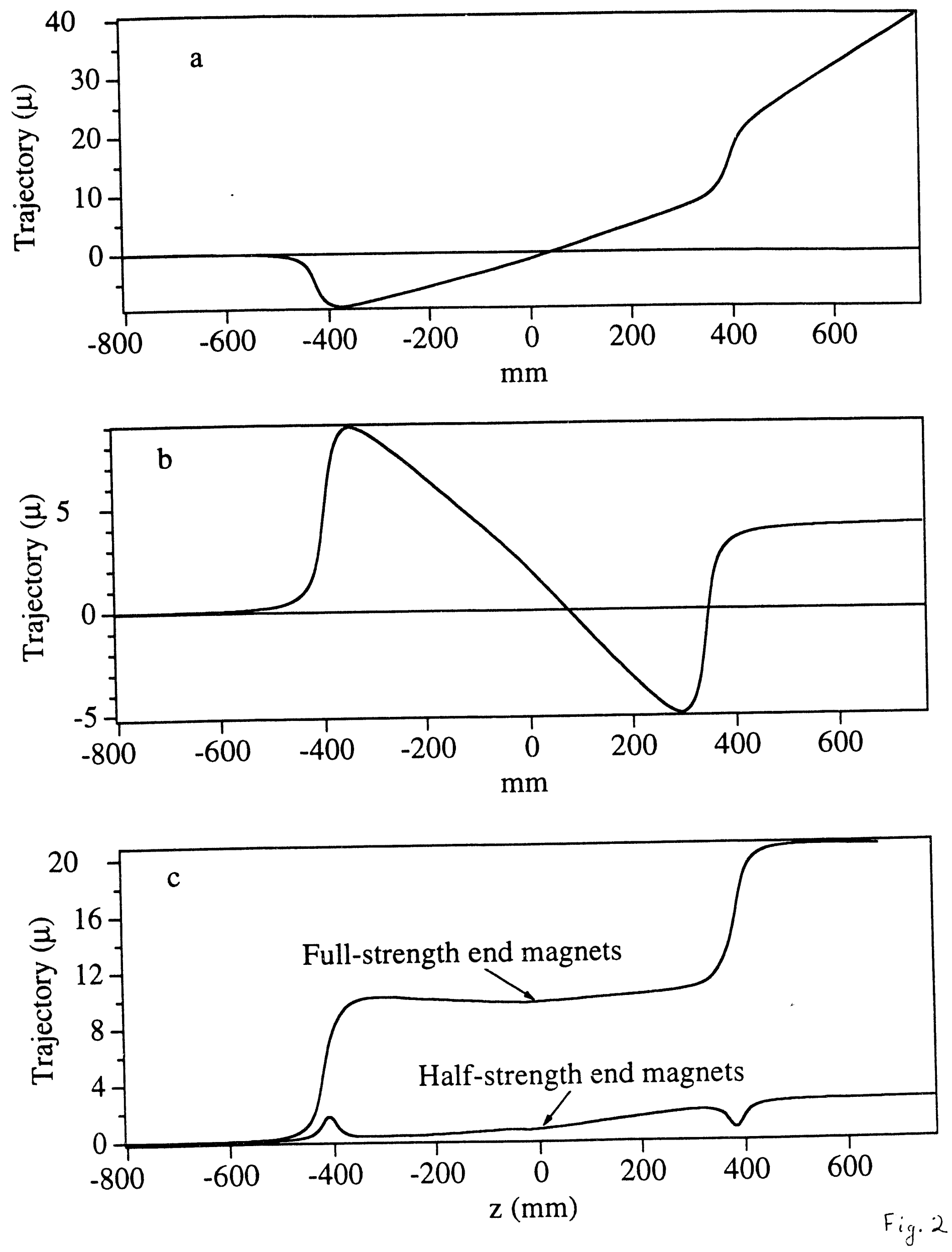

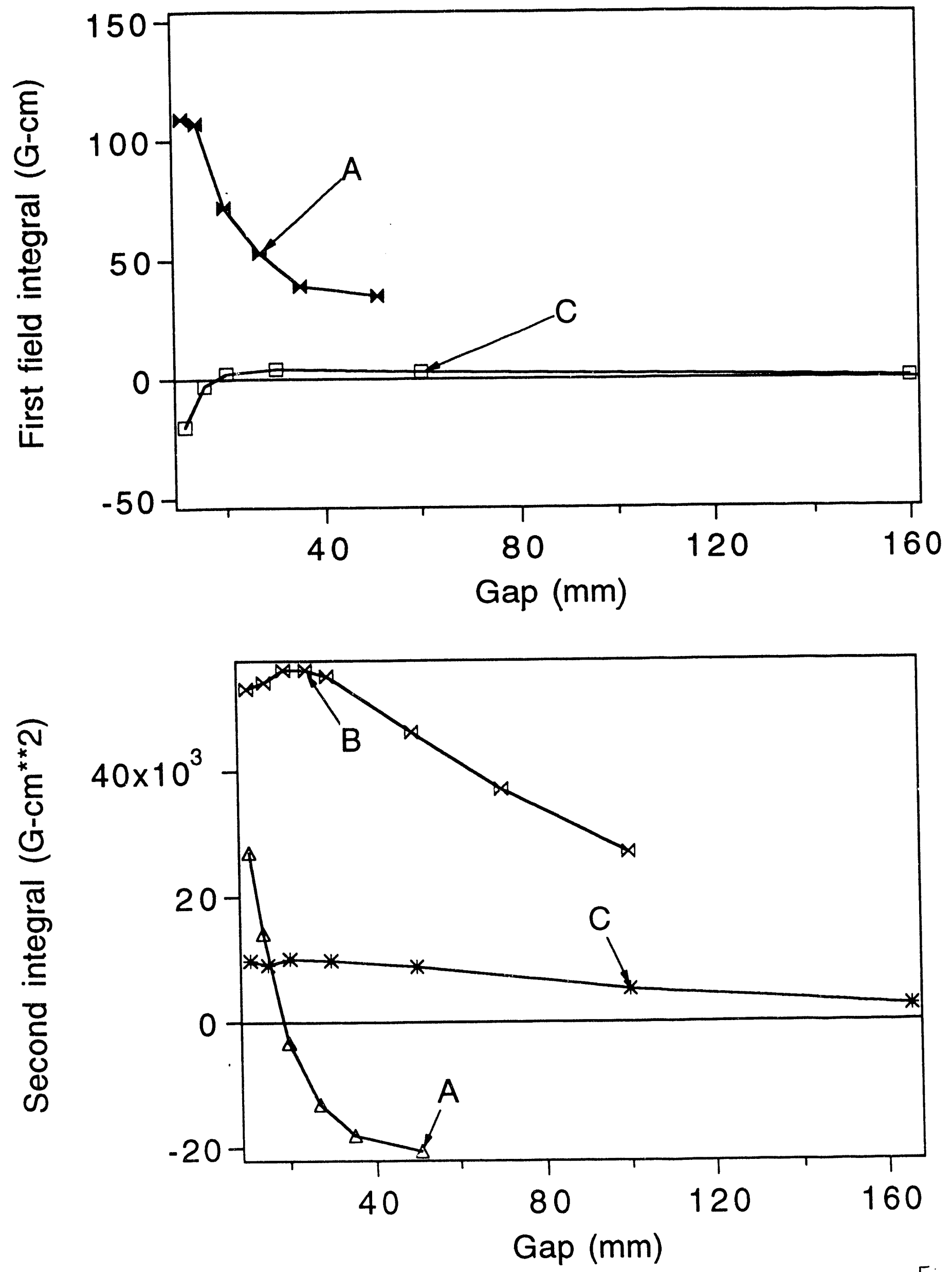

Fig. 3 


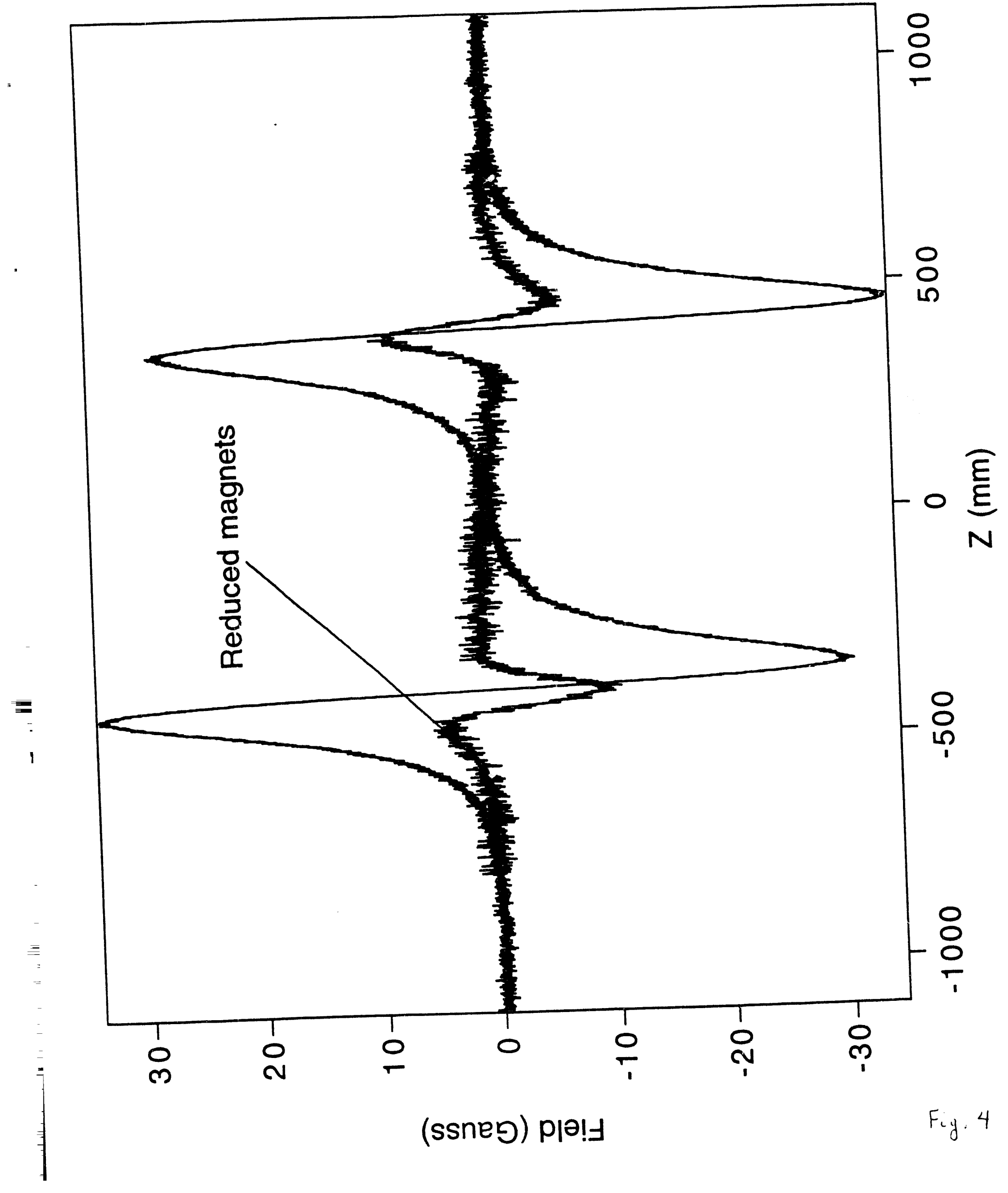



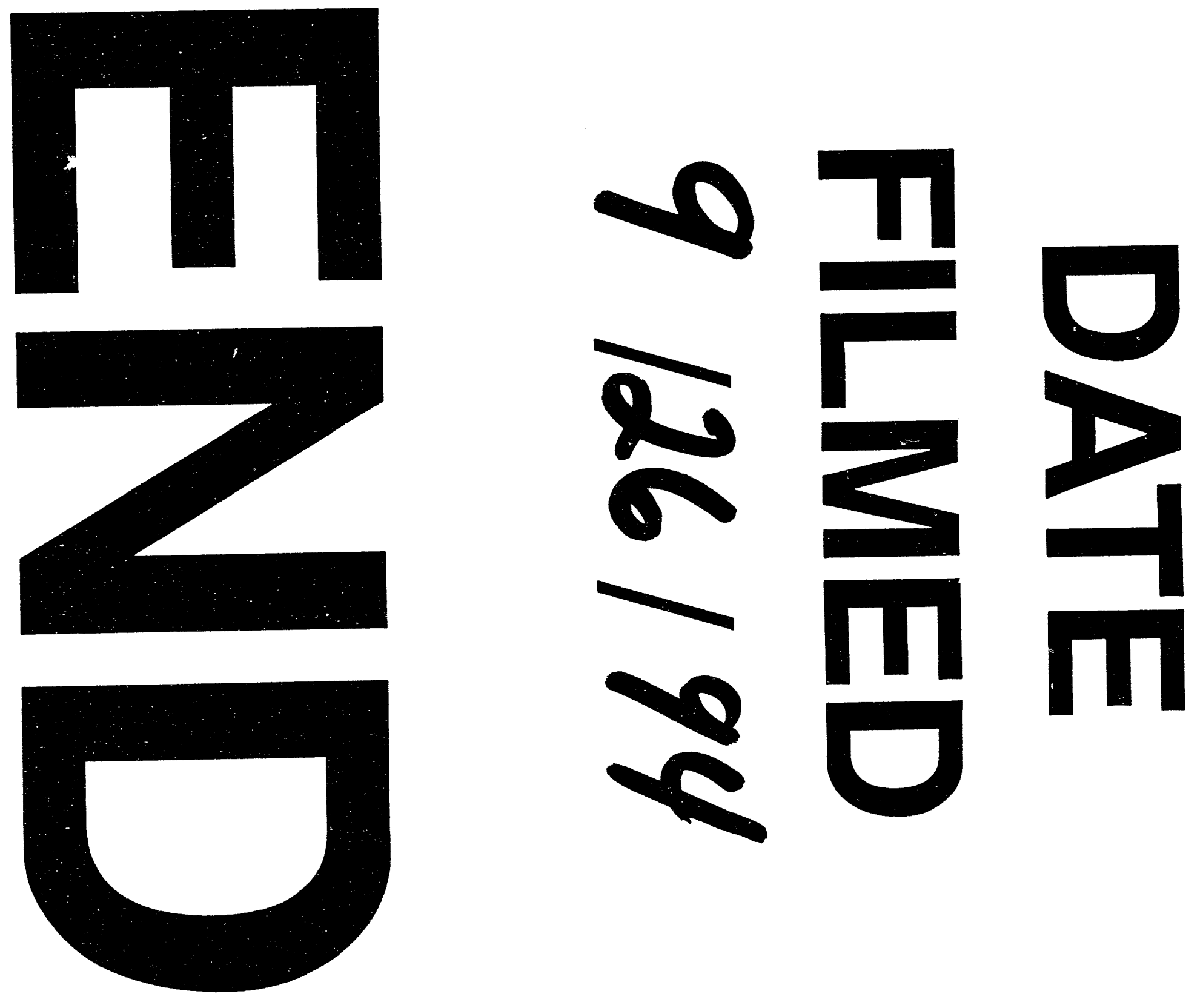


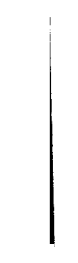

